\title{
The evidence for noncosmetic uses of botulinum toxin
}

\author{
Botulinum toxin has been studied for a variety of uses \\ beyond the cosmetic. Here's what you need to know \\ about which uses are worth considering for your patient.
}

\section{PRACTICE RECOMMENDATIONS \\ > Do not use botulinum toxin for episodic migraine, tension headache, or cluster headaches. B \\ $>$ Consider off-label use of botulinum toxin for select patients with occipital and trigeminal neuralgia, gastroparesis, vaginismus, benign prostatic hypertrophy, neonatal brachial plexus palsy, post-stroke spasticity, and hemifacial spasm. (B) \\ $>$ Consider the use of botulinum toxin as an adjunct in chronic low back pain management. B}

Strength of recommendation (SOR) (A) Good-quality patient-oriented
evidence

(B) Inconsistent or limited-quality patient-oriented evidence

C Consensus, usual practice, opinion, disease-oriented evidence, case series
$\mathrm{M}$ ention the word "botulinum toxin" and one's mind is likely to go to the big business of cosmetic procedures. Among the 15.7 minimally invasive cosmetic procedures performed in 2017 , botulinum toxin type A (BoNT-A) made up the largest share, with 7.23 million procedures. ${ }^{1}$ However, botulinum toxin-which was first recognized for the ability to paralyze muscles through decreased release of acetylcholine-also has many pain-related and noncosmetic uses; some are approved by the US Food and Drug Administration (FDA) and others are off-label (see TABLE 1 $^{2-31}$ ). This review provides an evidence-based look at these uses, from those that have good evidence to support them-including chronic migraine and overactive bladder - to those that have limited (or no) evidence to support them-such as chronic pelvic pain and cluster headache.

But before we get into the evidence behind specific uses for botulinum toxin, let's review the available options and the potential risks they pose.

\section{Many options}

Although botulinum toxin is produced by Clostridium botulinum, the synthetic process to produce pharmaceuticals is patented and branded. BoNT-A is 1 of 7 recognized serotypes derived from $C$ botulinum; some examples of BoNT-A include onabotulinumtoxinA, abobotulinumtoxinA, and incobotulinumtoxinA. Clinically, the differences are minor, but they do allow for use of other brands if a patient becomes intolerant to the selected therapy. Treatment doses and costs for each brand vary.

Iraining. Primary care providers can obtain didactic training from pharmaceutical companies as well as skills training through workshops on botulinum toxin. Credentialed providers can perform some procedures in the primary care setting (TABLE 2).

Adverse effects also vary depending on the formulation and the sites injected. Patients generally tolerate the procedure
Blake Busey, DO, FAAFP; Mary Jo A. Esparza, DO Department of Family Medicine, Texas Tech University of Health Sciences El PasoTransmountain

Dblake.busey@ttuhsc.edu

The authors reported no potential conflict of interest relevant to this article.

doi: 10.12788/jfp.0101 
BoNT-A is 1 of 7 recognized serotypes derived from Clostridium botulinum.

TABLE 1

FDA-approved indications and off-label uses of botulinum toxin injections

\begin{tabular}{|c|c|}
\hline FDA-approved indications (SOR) & Off-label uses (SOR) \\
\hline $\begin{array}{l}\text { Beneficial } \\
\text { Chronic migraine with and without medication } \\
\text { overuse }(\mathbf{A})^{2-5} \\
\text { Blepharospasm }(\mathbf{A})^{5,25} \\
\text { Spasmodic dysphonia }(\mathbf{A})^{10} \\
\text { Cervical dystonia }(\mathbf{A})^{5,16} \\
\text { Limb spasticity }(\mathbf{A})^{31} \\
\text { Primary axillary hyperhidrosis }(\mathbf{A})^{11,12} \\
\text { Overactive bladder, idiopathic overactive } \\
\text { bladder, and neurogenic bladder }(\mathbf{A})^{28}\end{array}$ & 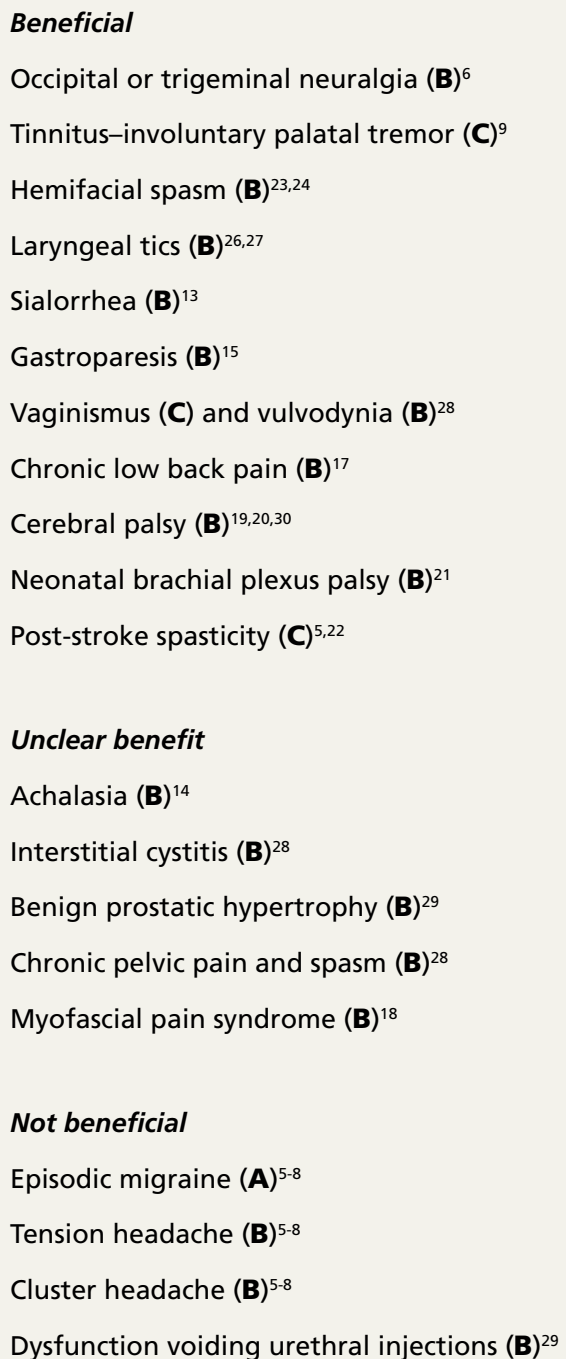 \\
\hline
\end{tabular}

FDA, Food and Drug Administration; SOR, strength of recommendation.

Strength of Recommendation (SOR)

A Good-quality patient-oriented evidence

B Inconsistent or limited-quality patient-oriented evidence

C Consensus, usual practice, opinion, disease-oriented evidence, case series

well, with discomfort from injections and localized bleeding as the major complaints. However, systemic events such as anaphylaxis and antibody development can occur. Depending on the formulation injected, the molecule can migrate and cause weakness in adjacent muscles, leading to undesired effects. Compensatory muscles can become strained, resulting in pain. Serious complications such as pneumonia and death have occurred with injection of botulinum toxin in or around the neck.

I A note about pain management. In addition to muscle relaxation, analgesic properties are among the identified benefits of BoNT-A injections. ${ }^{32,33}$ BoNT-A suppresses 
TABLE 2

Botulinum toxin-related procedures that are appropriate for primary care

\begin{tabular}{l|l|l}
\hline Condition & FDA approved? & Additional equipment needed \\
\hline Chronic migraine & Yes & None \\
\hline Cervical dystonia & Yes & Electromyography guidance recommended \\
\hline Blepharospasm & Yes & None \\
\hline Limb spasticity & Yes & Electromyography guidance recommended \\
\hline Post-stroke spasticity & No & Electromyography guidance recommended \\
\hline Occipital neuralgia & No & Consider ultrasound localization \\
\hline Hemifacial spasm & No & None \\
\hline Chronic low back pain & No & None \\
\hline
\end{tabular}

the release of norepinephrine, substance $\mathrm{P}$, and glutamate, which reduces pain sensitization. ${ }^{32}$ However, the extent of ongoing research involving BoNT-A uses in pain management exceeds the scope of this article. Some pain-related indications will be discussed, but the focus will be on other noncosmetic uses.

\section{Headache disorders}

I Chronic migraine affects $1.3 \%$ to $2.2 \%$ of the population and is defined as headaches occurring $\geq 15$ days ( $\geq 8$ migrainous days) per month. ${ }^{2}$ To qualify for BoNT-A treatment, patients must have tried 2 prophylactic medications that failed to provide relief, and their headaches must last at least 4 hours. Injections every 12 weeks with $5 \mathrm{U}$ in each of 31 prescribed sites is effective, as shown in the PREEMPT 2 study ${ }^{2}$ with external verification. ${ }^{3}$ The 24-week, double-blind, placebocontrolled study showed that BoNT-A treatment reduced headache days by 9 days $(P<.001)$ and migraine days by 8.7 days $(P<.001)^{2}$ and, at 108 weeks, injections reduced headache days by 10.7 days $(P<.0001))^{4,5}$

I Episodic migraine, tension headache, and cluster headaches. There is no significant BoNT-A-related pain reduction in episodic migraine $(\mathrm{n}=1838 ; 0.05$ headaches/mo; $95 \% \mathrm{CI},-0.26$ to -0.36 ) or tension headaches $(\mathrm{n}=675 ;-1.43$ headaches $/ \mathrm{mo}$; 95\% CI, -3.13 to -0.27$)^{5,6}$ For cluster headaches, a single prospective study with low enrollment showed no consistent benefit, ${ }^{7}$ while a pilot study showed some improvement, with reduction of attacks by $50 \%$ in half of subjects. ${ }^{8}$

I Occipital neuralgia and trigeminal neuralgia entail paroxysmal, brief, shocklike pain without associated deficits affecting the respective nerve distributions. Multiple prospective and double-blind placebo-controlled studies with relatively low enrollment show consistent improvement in pain intensity, number of pain-free days, analgesic consumption, and headache frequency with BoNT-A added to nerve blocks. ${ }^{6}$

\section{ENT disorders}

I Tinnitus by involuntary palatal tremor causes a discontinuous clicking noise. Palatal tremor can be treated with BoNT-A $15 \mathrm{U}$ to tensor veli palatini and levator veli muscles to provide temporary relief for 2 to 6 months. ${ }^{9}$

I Spasmodic dysphonia and voice tremor are the result of laryngeal hyperkinesis, and BoNT-A has been deemed the gold standard of treatment. BoNT-A is administered via bilateral injection of the thyroarytenoid muscles for patients with adductor-type spasmodic dysphonia and of the posterior cricoarytenoid muscles for those with the abductor type. A series of 1300 patients (predominantly with the adductor type) treated with BoNT-A showed a $100 \%$ improvement in symptoms for 6 to 15 weeks. Patients with abductor-type spasmodic dysphonia were
Patients generally tolerate the procedure well, with discomfort from injections and localized bleeding as the major complaints. 


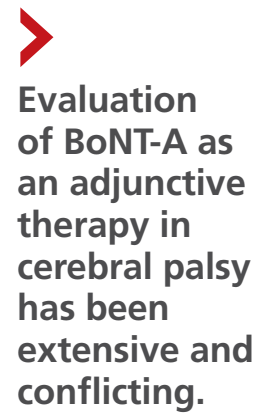

found to have $89 \%$ improvement in Voice Related Quality of Life Index score. ${ }^{10}$

\section{Secretory disorders}

- Primary axillary hyperhidrosis (PAH) is an idiopathic excessive production of sweat occurring for at least 6 months, typically with onset before age 25 years. PAH can cause significant psychosocial and physical impairment. Current treatments include topical aluminum chloride, systemic anticholinergics, and thoracic sympathectomy, which can provide temporary relief but are not well tolerated.

BoNT-A treatment is efficacious, safe, and improves quality of life for $\mathrm{PAH}$ patients. A 52-week, multicenter, double-blind, randomized, placebo-controlled study showed significant reductions in symptom severity, decreased sweating at rest by gravimetric testing, and improvements in self-reported quality of life. ${ }^{11}$ A 10-year retrospective study in patients ages 12 years and older showed a $75 \%$ to $100 \%$ improvement in hyperhidrosis, with a median treatment effect duration of 7 months. $^{12}$

- Sialorrhea, or hypersalivation, is typically associated with neurological conditions such as cerebral palsy, amyotrophic lateral sclerosis, Parkinson disease, and posttraumatic brain injuries. It typically is treated with anticholinergic drugs, surgery, and irradiation of salivary glands, which can have significant adverse effects and complications. In a randomized blinded study, BoNT-A injections in the parotid and submandibular glands resulted in a dramatic reduction of sialorrhea and were safe and well tolerated..$^{13}$

\section{Gastric disorders}

- Achalasia is a syndrome of aperistalsis and incomplete lower esophageal sphincter (LES) relaxation with a "bird beak" appearance on barium swallow. Patients who meet diagnostic criteria are treated with pneumatic dilation or myotomy; however, some patients demonstrate symptoms of achalasia but don't meet the diagnostic criteria. In these patients, BoNT-A injection in the LES provides symptomatic relief. In a case series,
LES BoNT-A injections $20 \mathrm{U}$ were used as a decision tool in whether to proceed with definitive treatment. ${ }^{14}$

Gastroparesis is a disorder of impaired gastric motility without mechanical obstruction. Pyloric sphincter BoNT-A injections are useful in refractory patients. Multiple prospective, noncontrolled (4), retrospective (3), and randomized placebo-controlled (2), studies with limited enrollment showed benefit for $37.5 \%$ to $100 \%$ of patients receiving BoNT-A injections of 80 to $200 \mathrm{U.}^{15}$

\section{Musculoskeletal disorders}

- Cervical dystonia (CD) entails involuntary contractions of the neck and upper shoulder musculature, causing abnormal neck, shoulder, and head posturing. BoNT-A is first-line treatment for CD. ${ }^{5}$ BoNT-A is more efficacious than trihexyphenidyl based on multiple large, high-quality studies. ${ }^{16}$

Chronic low back pain (CLBP) is defined as back pain persisting $\geq 12$ weeks. More than $80 \%$ of adults have had at least 1 episode of back pain in their lifetime. A 14-month open-label, pilot study evaluating the short- and long-term effects of paraspinal muscle BoNT-A injections for refractory CLBP showed reduced pain intensity, reduced number of pain days, and functional improvements. ${ }^{17}$

Myofascial pain syndrome (MPS) consists of myofascial trigger points (palpable, tender nodules that produce pain) with multiple pathophysiological etiologies that include dysfunctional acetylcholine activity, which releases nociceptive neurotransmitters. Studies have yielded inconsistent effects of BoNT-A on MPS. ${ }^{18}$

\section{Spastic disorders}

- Cerebral palsy (CP) involves altered muscle tone, posture, and movement secondary to central motor dysfunction with spasticity. Evaluation of BoNT-A as an adjunctive therapy in CP has been extensive and conflicting. A prospective cohort study evaluating gastrocsoleus BoNT-A injections along with gait analysis in 37 children with CP showed no significant improvements. ${ }^{30}$ In 60 children with 
$\mathrm{CP}$ who received BoNT-A injections, there was improvement in muscle tone and range of motion, while gait improved in patients up to (but not after) age 7 years..$^{19} \mathrm{~A}$ multicenter Dutch study of 65 children compared BoNT-A injections in addition to a comprehensive rehabilitation program vs rehabilitation alone, with no difference identified. ${ }^{20}$

I Neonatal brachial plexus palsy (NBPP) is damage to the brachial plexus as a result of trauma during the perinatal period. It is typically self-resolving but can cause residual functional impairment. Surgery is recommended for serious injuries or if functional recovery is not achieved within 9 months. Off-label use of BoNT-A has been shown to be effective in relieving muscle contractures and imbalance, but data are limited and there have only been small studies performed. ${ }^{21}$ A retrospective cohort study of 59 patients with NBPP who received BoNT-A injections showed improved range of motion and function of the affected extremity. Moreover, surgical intervention was deferred, modified, or averted in patients who were under consideration for more invasive treatment. ${ }^{21}$

I Post-stroke spasticity can be temporarily relieved with the use of BoNT-A injections. Several studies have examined the effect of BoNT-A coupled with rehabilitation programs vs injections alone in the treatment of post-stroke spasticity. Devier et al found that improvements in spasticity scores did not differ between groups; however, implementing rehabilitation after BoNT-A injections was associated with improved function compared to injection alone. ${ }^{31}$ A 2018 randomized, double-blind, placebo-controlled trial demonstrated improvements in both treatment groups: those who received BoNT-A plus targeted rehab regimen and those who received saline injection plus rehab. ${ }^{22}$ In this case, it appears BoNT-A acts as more of an adjunct to physical therapy in the treatment of post-stroke spasticity. ${ }^{5}$

I Hemifacial spasm is an involuntary, brief, irregular unilateral (sometimes bilateral) spasm of the face in the distribution of the facial nerve. Injections with BoNT-A have been deemed effective by the American Academy of Neurology. ${ }^{23}$ A 16 -year retrospective study examined the efficacy and adverse effects of BoNT-A in the treatment of hemifacial spasm in 113 patients with a mean age of 63.1 years; it demonstrated high efficacy and mild temporary adverse effects. ${ }^{24}$ The duration of improvement averaged 16 weeks; pretarsal injections had better results than preseptal injections; and there were no differences between the commercial brands.

I Blepharospasm is a focal dystonia marked by excessive blinking and involuntary eye closures due to overexcitability of orbicularis oculi and periocular muscles, and BoNT-A is the treatment of choice. ${ }^{5,25} \mathrm{~A}$ retrospective review of 19 patients with blepharospasm who were treated with BoNT-A for more than 5 years found that BoNT-A is a stable and effective treatment with an adverse event rate of $4 \%$. Additionally, there were no differences found in clinical efficacy between the 4 BoNT-A brands on the market. ${ }^{25}$

I Laryngeal tics can cause significant psychosocial distress for patients. This condition is characterized by involuntary, recurrent rhythmic sounds that are often preceded by premonitory urges that are relieved by the behavior. An open-label, uncontrolled, confirmatory study with 30 subjects showed that bilateral vocal cord BoNT-A injections resulted in $93 \%$ improvement in vocal tics. ${ }^{26} \mathrm{~A}$ subsequent study highlighted case histories of 2 patients with laryngeal tics who received thyroarytenoid muscle BoNT-A injections and had marked reduction in symptoms and premonitory sensations. ${ }^{27}$ Although these small studies have suggested possible effectiveness of BoNT-A for laryngeal tics, there is no high-quality evidence.

\section{Urologic disorders}

I Overactive, idiopathic overactive, or neurogenic bladder causes increased urinary frequency, urgency, and nocturia without infectious etiology; they can be a result of neurologic dysregulation, detrusor overactivity, or idiopathic causes. Intravesical BoNT-A injection of 100 to $300 \mathrm{U}$ has been found effective for symptoms refractory to anticholinergic and lifestyle therapy, with increased cystometric capacity (229.1 to $427 \mathrm{~mL}$, $P<.00001)$, decreased maximum detrusor pressure (60.7 to $26.1 \mathrm{~cm} \mathrm{H} 2 \mathrm{O}, P<.00001)$,

\section{$>$}

It appears

BoNT-A acts as more of an adjunct to physical therapy in the treatment of post-stroke spasticity. 


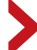

\section{Intravesicular}

BoNT-A

injections have

not consistently

been effective

in the treatment

of interstitial

cystitis. and resolution of urgency in $87 \%$ of patients $(P<.001) .^{28}$

Interstitial cystitis, also known as painful bladder syndrome, is characterized by reduced bladder emptying, urethral pressure, and residual urine pressure, with symptoms of increased urinary frequency without infection. Intravesicular BoNT-A injections have not consistently been effective in treatment of this condition. ${ }^{28}$

Dysfunctional voiding, urethral sphincter overactivity, and Fowler syndrome involve urethral sphincter spasticity with difficulty passing urine and possibly retention. Urethral sphincter injections of $100 \mathrm{U}$ BoNT-A improved flow rates and decreased residual volume. A randomized, doubleblinded, placebo-controlled study showed a significantly improved International Prostate Symptom Score (IPSS), quality of life index, maximum flow rate, voided volume, and decreased detrusor voiding pressure at 1 month. ${ }^{29}$

Benign prostatic hypertrophy (BPH) is a very common condition leading to outlet obstruction. The mainstays of treatment are 5- $\alpha$-reductase inhibitors, $\alpha$-adrenergic blockers, and surgical removal. Intraprostatic BoNT-A injections of 100 to $200 \mathrm{U}$ were initially promising, and subsequent randomized, double-blind, placebo-controlled studies demonstrated patients with moderate-tosevere symptoms (IPSS $\geq 19$ ) had improved IPSS, maximum flow rate, and post-void residual volume compared to placebo. ${ }^{29}$

\section{Gynecologic disorders}

- Vaginismus is the involuntary, recurrent, or persistent contraction of the perineal muscles surrounding the outer third of the vagina; it is classified by 4 progressively more severe degrees of intensity. Levator ani, bulbospongiosus, bulbocavernosus, pubococcygeus, and/ or puborectalis muscle BoNT-A injections have shown benefits in decreasing resistance to vaginal exams (95.8\%) and the ability to achieve satisfactory sexual intercourse after first injection (75\%-100\%). Effects were transient for up to $15.4 \%$ of patients requiring repeat injections. $^{28}$

Vulvodynia is vulvar pain and orgas- mic difficulties and has been treated with bulbospongiosus muscle BoNT-A injections in retrospective studies. A single randomized, double-blinded, placebo-controlled study showed significantly improved pain scores after 1 to 2 injection series. ${ }^{28}$

-Chronic pelvic pain is a syndrome of somatic functional or regional pain, which can be caused by the spasm of the pelvic musculature with or without trigger points. Patients with pain refractory to treatment have been treated with levator ani injections. A retrospective cohort study found $79.3 \%$ of patients experienced pain relief and $20.7 \%$ reported improved symptoms. In a doubleblind, randomized, placebo-controlled trial, pelvic floor muscles were injected with $80 \mathrm{U}$ BoNT or saline, and symptoms were evaluated along with vaginal manometry. BoNT was associated with a reduction in some pain but not as much as placebo, while vaginal pressures decreased more with BoNT than with placebo. $^{28}$

JFP

CORRESPONDENCE

Blake Busey, DO, FAAFP, Texas Tech University of Health Sciences El Paso-Transmountain, 2000B Transmountain Road, Suite B400, El Paso, TX 79911; blake.busey@ttuhsc.edu

\footnotetext{
REFERENCES

1. American Society of Plastic Surgeons. New statistics reveal the shape of plastic surgery [news release]. March 1, 2018. www. plasticsurgery.org/news/press-releases/new-statistics-revealthe-shape-of-plastic-surgery. Accessed October 23, 2020.

2. Diener HC, Dodick DW, Aurora SK, et al; PREEMPT 2 Chronic Migraine Study Group. OnabotulinumtoxinA for treatment of chronic migraine: results from the double-blind, randomized, placebo-controlled phase of the PREEMPT 2 trial. Cephalgia. 2010;30:804-814.

3. Herd CP, Tomlinson CL, Rick C, et al. Botulinum toxins for the prevention of migraine in adults. Cochrane Database Syst Rev. 2018;6:CD011616.

4. Blumenfeld AM, Stark RJ, Freeman MC, et al. Long-term study of the efficacy and safety of OnabotulinumtoxinA for the prevention of chronic migraine: COMPEL study. J Headache Pain. 2018;19:13.

5. Simpson DM, Hallett M, Ashman EJ, et al. Practice guideline update summary: botulinum neurotoxin for the treatment of blepharospasm, cervical dystonia, adult spasticity, and headache: report of the Guideline Development Subcommittee of the American Academy of Neurology. Neurology. 2016;86:1818-1826.

6. Luvisetto S, Gazerani P, Cianchetti C, et al. Botulinum toxin type A as a therapeutic agent against headache and related disorders. Toxins. 2015;7:3818-3844.

7. Sostak P, Krause P, Förderreuther S, et al. Botulinum toxin typeA therapy in cluster headache: an open study. J Headache Pain. 2007;8:236-241.

8. Bratbak DF, Nordgård S, Stovner LJ, et al. Pilot study of sphenopalatine injection of onabotulinumtoxinA for the treatment of intractable chronic cluster headache. Cephalalgia. 2016;36: 503-509.

9. Mandavia R, Dessouky O, Dhar V, et al. The use of botulinum toxin in otorhinolaryngology: an updated review. Clin Otolaryngol. 2014;39:203-209.
} 
10. Klein AM, Stong BC, Wise J, et al. Vocal outcome measures after bilateral posterior cricoarytenoid muscle botulinum toxin injections for abductor spasmodic dysphonia. Otolaryngol Head Neck Surg. 2008;139:421-423.

11. Naumann M, Lowe NJ. Botulinum toxin type A in treatment of bilateral primary axillary hyperhidrosis: randomised, parallel group, double blind, placebo controlled trial. BMJ. 2001;323: 596-599.

12. Rosen R, Stewart T. Results of a 10-year follow-up study of botulinum toxin A therapy for primary axillary hyperhidrosis in Australia. Intern Med J. 2018;48:343-347.

13. Restivo DA, Panebianco M, Casabona A, et al. Botulinum toxin A for sialorrhoea associated with neurological disorders: evaluation of the relationship between effect of treatment and the number of glands treated. Toxins (Basel). 2018;10:55.

14. Katzka DA, Castell DO. Use of botulinum toxin as a diagnostic/therapeutic trial to help clarify an indication for definitive therapy in patients with achalasia. Am J Gastroenterol. 1999;94 637-642.

15. Ukleja A, Tandon K, Shah K, et al. Endoscopic botox injections in therapy of refractory gastroparesis. World J Gastrointest Endosc. 2015;7:790-798.

16. Zakin E, Simpson D. Evidence on botulinum toxin in selected disorders. Toxicon. 2018;147:134-140.

17. Jabbari B, Ney J, Sichani A, et al. Treatment of refractory, chronic low back pain with botulinum neurotoxin A: an open-label, pilot study. Pain Med. 2006;7:260-264.

18. Climent JM, Kuan TS, Fenollosa P, et al. Botulinum toxin for the treatment of myofascial pain syndromes involving the neck and back: a review from a clinical perspective. Evid Based Complement Alternat Med. 2013;2013:381459.

19. Mirska A, Cybula K, Okurowska-Zawada B, et al. Use of botulinum toxin in the treatment of ankle plantar flexor spasticity in children with cerebral palsy. J Pediatr Orthop B. 2014;23:517-522.

20. Schasfoort F, Pangalila R, Sneekes EM, et al. Intramuscular botulinum toxin prior to comprehensive rehabilitation has no added value for improving motor impairments, gait kinematics and goal attainment in walking children with spastic cerebral palsy. Rehabil Med. 2018;50:732-742.

21. Michaud LJ, Louden EJ, Lippert WC, et al. Use of botulinum toxin type A in the management of neonatal brachial plexus palsy. PM R. 2014;6:1107-1119.

22. Prazeres A, Lira M, Aguiar P, et al. Efficacy of physical therapy associated with botulinum toxin type A on functional performance in post-stroke spasticity: A randomized, double-blinded, placebo-controlled trial. Neurol Int. 2018;10:7385.

23. Simpson DM, Blitzer A, Brashear A, et al; Therapeutics and Technology Assessment Subcommittee of the American Academy of Neurology. Assessment: Botulinum neurotoxin for the treatment of movement disorders (an evidence-based review): report of the Therapeutics and Technology Assessment Subcommittee of the American Academy of Neurology. Neurology. 2008;70:1699-1706.

24. Sorgun MH, Yilmaz R, Akin YA, et al. Botulinum toxin injection for the treatment of hemifacial spasm over 16 years. JClin Neurosci. 2015;22:1319-1325.

25. Lee S, Park S, Lew H. Long-term efficacy of botulinum neurotoxin-A treatment for essential blepharospasm. Korean J Ophthalmol. 2018;32:1-7.

26. Porta M, Maggioni G, Ottaviani F, et al. Treatment of phonic tics in patients with Tourette's syndrome using botulinum toxin type A. Neurol Sci. 2004;24:420-423.

27. Vincent DA Jr. Botulinum toxin in the management of laryngeal tics. J Voice. 2008;22:251-256.

28. Moga MA, Dimienescu OG, Balan A, et al. Therapeutic approaches of botulinum toxin in gynecology. Toxins (Basel) 2018;10:169.

29. Jhang J-F, Kuo H-C. Novel applications of onabotulinumtoxinA in lower urinary tract dysfunction. Toxins (Basel). 2018;10:260.

30. Hastings-Ison T, Sangeux M, Thomason P, et al. Onabotulinum toxin-A (Botox) for spastic equinus in cerebral palsy: a prospective kinematic study. J Child Orthop. 2018;12:390-397.

31. Devier D, Harnar J, Lopez L, et al. Rehabilitation plus onabotulinumtoxina improves motor function over onabotulinumtoxina alone in post-stroke upper limb spasticity: a single-blind, randomized trial. Toxins. 2017:9:216.

32. Sim WS. Application of botulinum toxin in pain management Korean J Pain. 2011;24:1-6.

33. Safarpour Y, Jabbari B. Botulinum toxin treatment of pain syndromes-an evidence based review. Toxicon. 2018;147: 120-128.

\section{(1) Visit us @ \\ mdedge.com/familymedicine}

(1) COVID-19 treatment: What the NIH recommends

Doug Campos-Outcalt, MD, MPA

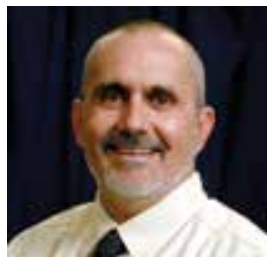

(1) Study offers reassurance to postmenopausal women taking hormone therapy

(1) Tackling unhealthy substance use using USPSTF guidance and a 1-question tool

(1)) Medication use \& COVID-19: Unwarranted concerns, evidence-based approaches

\section{1) 3 new latent TB preventive regimens} dramatically cut Tx time

\section{ONLINE EXCLUSIVES}

\section{- RESIDENTS' RAPID REVIEW}

A 5-question monthly quiz to help you prepare for the family medicine (re)certification exam. This month: Obesity

\section{PHOTO ROUNDS FRIDAY}

Test your diagnostic skills at www.mdedge.com/ familymedicine/photo-rounds

\section{PLUS}

Today's headlines in family medicine
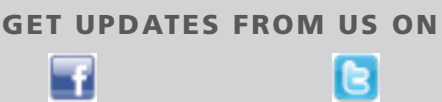

www.facebook.com/JFamPract

http://twitter.com/JFamPract

www.mdedge.com/familymedicine 